Stefaniya Ptashnyk

\title{
Sprachengebrauch und Sprachenwechsel an der Lemberger Universität im ausgehenden 18. und in der ersten Hälfte des 19. Jahrhunderts
}

\section{Vorbemerkungen}

In seinen Jubiläumserinnerungen anlässlich des 250jährigen Bestehens der Universität Lemberg im Jahre 1911 schrieb der polnische Historiker und Publizist Franciszek Jaworski Folgendes:

W ogniu walki poczęta, w walce półtrzecia trwa wieku Alma Mater lwowska. ... Jest potężną twierdzą ducha polskiego, czujną strażnicą u wschodnich rubieży Polski, jest jasnem ogniskiem wiedzy, prawdy i kultury, dostojną siedzibą nauki. (Jaworski 1912: 1)

Im Feuer der Kämpfe geboren, besteht sie seit 250 Jahren im Kampf, die Lemberger Alma Mater. Sie ist eine mächtige Festung des polnischen Geistes, ein wachsamer Außenposten an den östlichen Grenzen Polens, helle Flamme der Wissens, der Wahrheit und der Kultur, eine würdevolle Stätte der Wissenschaft. [dt. Übersetzung: S. P.]

Jaworski hebt in seinen Ausführungen ganz prominent den polnischen Nationalcharakter der Universität Lemberg hervor sowie ihre Rolle für die polnische Wissenschaft. Von den anderen Kulturen und Nationalitäten ist hier, in diesem Text von 1911/12, keine Rede.

Patriotisch geprägt, aber nicht national festgelegt waren hingegen die Töne, die im Jahr 1784, d. h. dem Jahr der Neugründung der Universität durch den Kaiser Joseph II., erklangen. Die feierlichen Inaugurationsakten vom 6. November 1784 - allesamt auf Lateinisch verfasst - wurden zwei Jahre später, also 1786, in der Druckerei von Thomas Piller in Lemberg abgedruckt und als Anhang sind den Akten auf Deutsch gedichtete „Oden über die Einführung der hohen Schule zu Lemberg“ beigefügt. Hier ist zwar an mehreren Stellen vom „Volk“ die Rede, nationale Züge hat dieses „Volk“ in der Ode jedoch nicht:

Groß muß ewiger Lohn jenes Monarchens seyn,

Der wie Joseph sein Volk Weißheit gelehret hat:

Stefaniya Ptashnyk, Heidelberger Akademie der Wissenschaften, Karlstr. 4, 69117 Heidelberg, E-Mail: stefaniya.ptashnyk@adw.uni-heidelberg.de

Ә Open Access. (C) 2018 Stefaniya Ptashnyk, publiziert von De Gruyter. (c) BY-NC-ND Dieses Werk ist lizenziert unter der Creative Commons Attribution-NonCommercial-NoDerivatives 4.0 Lizenz. 
Groß auch seye der Dank, Söhne von Halicz, Ihm!

Lieben Joseph den Vater des Volks! (aus: Acta Universitatis ... 1786: 61) ${ }^{1}$

In der Zeit zwischen den beiden genannten Publikationen erlebte die Universität Lemberg eine wechselvolle Geschichte, vor allem im Hinblick auf die sprachliche Prägung. Dieser „Sprachgeschichte“ ist nun der vorliegende Beitrag gewidmet, und ich möchte dabei den Sprachengebrauch sowie den Sprachenwechsel an der Universität Lemberg eingehender untersuchen. Der Hauptfokus wird auf der Zeit der so genannten Josephinischen Universität und ihrer Nachfolgerinnen liegen, d. h. von 1784 an bis in die 1870er Jahre hinein. Diese Zeitspanne ist insofern besonders interessant, als hier die Koexistenz des Lateinischen, Deutschen und anderer Sprachen im universitären Bereich aufgezeigt werden kann. Besondere Berücksichtigung gilt den Unterrichtssprachen.

Als Quellen für die Untersuchung dienen mir vor allem die Vorlesungsverzeichnisse der Universität Lemberg, die teils im Gebietsarchiv Lviv, teils in den österreichischen Bibliotheken ${ }^{2}$ verfügbar sind. Aus praktischen Gründen muss die Auswahl der Textsorten für die Analyse eingeschränkt bleiben.

Von Anfang an wirkte die Universität Lemberg in einem multilingualen, multikonfessionellen und multikulturellen Raum. Um an dieser Stelle ein ungefähres Bild von den in Lemberg lebenden Nationalitäten zu vermitteln, möchte ich die uns bekannten statistischen Daten aus dem untersuchten Zeitraum anführen. Dabei ist anzumerken, dass für die Zeit vor 1850 nur sehr wenige Informationen über die Bevölkerungszusammensetzung von Lemberg vorliegen. ${ }^{3}$ In einem Bericht an die Lemberger Polizeibehörde nennt der galizische

1 Die Josephinische Universität wurde als eine deutschsprachige Institution gegründet. Die feierliche Inauguration, die am 16. November 1784 stattfand, folgte jedoch der Tradition der akademischen Feiern und wurde auf Lateinisch abgehalten (vgl. Finkel \& Starzyński 1894: 58). Davon zeugt die erwähnte Sammlung der Inaugurationsreden, die zwei Jahre später (1786) in der Druckerei von Thomas Piller in Lemberg unter dem Titel „Acta Vniversitatis Leopolitanae in Galicia Anno MDCCLXXXIV. Inauguratae“ gedruckt wurde. Neben den in Latein verfassten Festreden sind in diesem Band später entstandene Oden auf die Universität, die Bildung und den Kaiser enthalten, die sowohl lateinisch als auch deutsch gedichtet sind. Sie stammen aus der Feder des Obergerichtrates Ritter von Rittershayn und der Professoren van Swieten und Brygida.

2 Für die Unterstützung in der Materialeinsicht geht an dieser Stelle mein großer Dank an Georg Schratteneger, Mitarbeiter der Salzburger Universitätsbibliothek, in der sich - neben den Wiener Bibliotheken - gut erhaltene Bestände der Vorlesungsverzeichnisse befinden.

3 Für das späte 19. Jahrhundert sieht die Lage anders aus. Ab 1880 wurden in der Monarchie regelmäßig Volkszählungen durchgeführt. Dank ihrer Ergebnisse bekommen wir deutlich differenziertere Informationen über die Lemberger Kontaktsprachen und ihre Sprecher. Für das Jahr 1910 vgl. z. B. Österreichische Statistik (1912: 63 und 80-81). 
Statthalter Agenor Graf Gołuchowski folgende Bevölkerungszahlen für das Jahr 1851: Einwohner insgesamt - 68.835, davon römisch-katholisch - 33.224, griechisch-uniert - 4.090, Juden - 21.357, „,sonstige“ - 626. Die Einwohner umfassten 6.000 Deutsche und 28.000 Polen; schließlich gab es noch 9.538 „Fremde“ (vgl. Die Ruthenische Sprach- und Schriftfrage 1861: 246f.).

Für das Verständnis der sprachlich-ethnischen Besonderheiten Lembergs sind zum Teil die Angaben zur Konfession der Bevölkerung hilfreich: Der Anteil der Jiddisch sprechenden Bevölkerung lässt sich anhand ihres Bekenntnisses zur mosaischen (alias jüdischen oder israelitischen) Religion ermitteln. 1851 machen die Juden ca. ein Drittel der Stadtbevölkerung aus. In dieser Gruppe kann man das Jiddische in der privaten Kommunikation erwarten, auch wenn nicht in jeder sozialen Schicht. Ferner beherrschen die gebildeten Juden das Hebräische. Die Römischen Katholiken sprachen im Alltag meist Polnisch, die Unierten Katholiken waren hingegen überwiegend Ruthenen. Nach den aufgeführten Zahlen dürfte mehr als die Hälfte der Lemberger Polnisch gesprochen haben, die Ruthenen betrugen weniger als $10 \%$ der Gesamtbevölkerung. Die Deutschsprachigen konnten sowohl römisch-katholisch als auch evangelisch sein und ihre Zahl betrug ca. 9\% der Gesamtbevölkerung.

Man sprach in Lemberg also Polnisch, Ukrainisch (Ruthenisch), ${ }^{4}$ Deutsch und Jiddisch. In dieser Statistik nicht erfasst, aber durch andere Quellen belegt sind Armenisch, Hebräisch oder Kirchenslawisch, die ebenfalls im Lemberg der Habsburger Zeit in Gebrauch waren. Und nicht zu vergessen - Latein. Die lateinische Sprache, die als Unterrichtssprache in vielen Schuleinrichtungen vorherrschte, war über einen längeren Zeitraum das verbindende Glied im Bereich Bildung für alle Nationalitäten.

\section{Die Gründungsgeschichte der Universität Lemberg}

Die Gründungsgeschichte der Lemberger Universität geht auf die Tätigkeit des Jesuitenordens zurück, der sich für die Eröffnung von öffentlichen Schulen einsetzte und in der Stadt das sogenannte Kollegium der Jesuiten gründete.

4 Das Ukrainische, das in den Gebieten der der k.u.k.-Monarchie gesprochen wurde, wird in den Quellen der österreichischen Periode meist als „Ruthenisch“ bezeichnet. Dabei handelt es sich um eine historische Varietät der heutigen ukrainischen Sprache, die auf der Basis der regional gesprochenen Mundarten in den westukrainischen Gebieten entstand und ca. 1820 zur Schriftsprache elaboriert wurde. Im Weiteren verwende ich die Bezeichnungen Ukrainisch und Ruthenisch in diesem Zusammenhang synonym. 
Das Kollegium (auch Jesuitenschule genannt) hatte schon $\mathrm{zu}$ Beginn des 17. Jahrhunderts alle Klassen einer mittleren humanistischen Schule mit dem Unterricht in Grammatik, Rhetorik, Poetik, Mathematik, Physik, Philosophie etc. So Łuszczak (2011):

Otwarte w 1608 roku we Lwowie szkoły publiczne posiadały już w 1609 roku wszystkie klasy średniej szkoły humanistycznej, a więc dwie klasy gramatyki, klasę syntaksy, poetyki i retoryki. Od 1611 roku rozpoczęto wykłady filozofii, a od 1613 roku matematyki i fizyki. (Łuszczak 2011: 44)

Eröffnet im Jahre 1608, besaßen die öffentlichen Schulen in Lemberg bereits 1609 alle Klassen einer mittleren humanistischen Schule, und zwar zwei Klassen Grammatik, je eine Klasse Syntax, Poetik und Rhetorik. 1611 wurde mit dem Philosophie-Unterricht begonnen und 1613 mit Mathematik und Physik. [dt. Übersetzung: S. P.]

Um das Jahr 1650 herum entstanden Pläne, das Kollegium der Jesuiten in eine Akademie umzuwandeln; aus diesem Grunde wurde das Lehrangebot um einen vierjährigen Theologie-Kurs erweitert (Łuszczak 2011: 44). Schließlich erfolgte auf der Basis des Kollegiums im Jahre $1661^{5}$ die Gründung der Hochschule unter dem Namen der „Akademie der Jesuiten“, bestätigt mit dem Gründungsdiplom des Königs Jan Kasimir. Der feierliche Gründungakt vom 20. Januar 1661 erklärte die Akademie bzw. die Universität zu Lemberg als gleichberechtigt in ihren Rechten und Privilegien mit der Jagiellonier Akademie zu Krakau.

Die Universität Lemberg hatte in den ersten Jahrhunderten ihrer Existenz eine durchaus verworrene Geschichte $\mathrm{zu}$ überstehen. Für manche Forscher stellt sich sogar die Frage, ob es sich bei dieser Hochschule um eine „Alma Mater Leopoliensis" handelt oder ob sie es mit etwa vier oder fünf Einrichtungen aus verschiedenen Phasen zu tun haben (vgl. z. B. Finkel \& Starzyński 1894). So lesen wir bei Dybiec (2011):

czy pod tą jednostkową, globalną nazwą Alma Mater Leopoliensis kryje się jedna uczelnia, czy też mamy do czynienia w poszczególnych okresach z kilkoma uniwersytetami czterema czy pięcioma? (Dybiec 2011: 66)

verbirgt sich hinter dem einheitlichen, globalen Namen Alma Mater Leopoliensis eine Bildungseinrichtung oder haben wir in verschiedenen Perioden mit verschiedenen Universitäten zu tun, etwa vier oder fünf? [dt. Übersetzung: S. P.]

5 Das Gründungsdiplom wurde jedoch vom Sejm nicht bestätigt. Der Grund bestand darin, dass die Krakauer Akademie keine Konkurrenz in Galizien dulden wollte. Somit waren gewisse Umwege erforderlich, um am Ende die Unterschrift des Königs zu organisieren. Dennoch gilt dieses Datum in der Geschichte der Universität Lemberg als ihr ursprüngliches Gründungsjahr (Euszczak 2011: 44). 
In den meisten historiografischen Arbeiten $\mathrm{zu}$ der Universität Lemberg/Lviv werden für die Zeit bis zum 1. Weltkrieg folgende Phasen hervorgehoben:

1. 1661-1784: Die Universität existiert unter dem Namen der Akademie der Jesuiten, welche auf der Basis der Jesuitenschulen durch das Privileg des Königs Jan Kasimir gegründet wurde. Latein ist zu dieser Zeit die vorwiegende Unterrichtssprache.

2. 1784-1805: In dieser Phase besteht die so genannte Josephinische Universität, deren „Neugründung“ durch den Kaiser Joseph II. erfolgte. In dieser Zeit beginnt der Übergang von der lateinischen Unterrichtssprache zu den „Volkssprachen“.

3. 1805-1817: Existenz als Lyzeum zu Lemberg.

4. 1817-1918: Franziszeische Universität.

Nach 1919 bestand die Universität unter dem Namen „Uniwersytet Jana Kazimierza“ und war überwiegend polnischsprachig (bis 1939), später firmierte sie als (ukrainische) „Ivan Franko-Universität“. Der heutige offizielle Name der Universität lautet seit 1999 „Lviver Nationale Ivan Franko-Universität“.

Die Universität bekam von Beginn an die Erlaubnis, in Theologie, Philosophie, Mathematik, Recht sowie in Medizin zu lehren; vgl. den Text des Privilegs:

Damus igitur potestatem, in eodem Leopoliensi Societatis Jesu Collegio, generale studium in omni facultate constituendi, theologiae nimirum tam scholasticae quam moralis, philosophiae, mathaeseos, iuris utriusque, medicinae, liberaliumque artium et disciplinarum, ac scientiarum omnino omnium, quascunque praedicti Patres Societatis Jesu tractandas ibidem per se vel per alios censueri rint, pro ipsorum arbitrio ac instituto, consuetoque àcademiarum, atque universitatum more et praxi. (abgedruckt in: Finkel \& Starzyński 1894: $20 \mathrm{f}$.

Wir verleihen somit die Macht, an dem erwähnten Kollegium der Lemberger Gesellschaft Jesu ein allgemeines Studium in allen Fakultäten einzurichten, selbstverständlich Theologie, sowohl scholastische als auch Moraltheologie, Philosophie, Mathematik, beiderlei Rechte, Medizin und freie Künste sowie überhaupt Disziplinen aller Wissenschaften, welche die oben erwähnten Väter der Gesellschaft Jesu ebenda selbst oder durch andere für angemessen zu lehren halten gemäß der eigenen Entscheidung und Ordnung, sowie gemäß der akademischen, universitären Gewohnheit und Praxis. [dt. Übersetzung: S. P.]

Als Verwaltungs- und Unterrichtssprache ist in den Anfängen vorwiegend Latein dokumentiert. Zugleich bekamen die Studierenden die Möglichkeit, mehrere moderne Sprachen zu erlernen (vgl. Finkel \& Starzyński 1894: 26).

Nach der ersten Teilung Polens 1772 befand sich die Lemberger Universität auf österreichischem Territorium. Ein Jahr später verließen die Jesuiten die Stadt infolge der Auflösung des Ordens (Wróblewski 2011: 271). Dies konnte an 
der betreffenden Institution nicht spurlos vorbeigehen. Da zu diesem Zeitpunkt das Bildungssystem und die Ausbildungsmethoden der Jesuiten an Ansehen verloren hatten, stand die Universität Lemberg nun vor der Notwenigkeit grundlegender Reformen (Finkel \& Starzyński 1894: 31). Das ausgehende 18. Jahrhundert war ohnehin die Zeit großer Bildungsreformen in der Monarchie, insofern erwies sich Lemberg samt dem Kronland Galizien als ein dankbares Experimentierfeld hierzu. Die Intention des Kaisers Joseph II. war, in Lemberg „eine wahre Universität und hohe Schule“ zu schaffen, ,allen übrigen Universitäten unserer Reiche und Länder gleich“ (Finkel \& Starzyński 1894: 52).

Am 21. Oktober 1784 wurde mit einem Diplom des Kaisers Joseph II. die deutschsprachige Lemberger Universität als Institution für höhere Bildung wiedergegründet. Diese Josephinische Universität war deutlich anders strukturiert als die bis dahin funktionierende Akademie der Jesuiten. Sie folgte dem Muster anderer europäischer Universitäten, die der Einmischung kirchlicher Strukturen entzogen und vollkommen dem Staat untergeordnet waren (Dybiec 2011: 67). Die Universität umfasste vier Fakultäten: Theologie, Jura, Medizin und Philosophie sowie ein vollständiges Gymnasium. Die Universität bekam das Recht der Promotion, Vergabe der Diplome für Doktoren, Lizentiaten und Magister an allen Fakultäten. Die Vorlesungen an der neu eröffneten Hochschule begannen am 17. November 1784.

In dieser Verfassung existierte die Lemberger Universität bis zum Beginn des 19. Jahrhunderts. Als 1795 infolge der 3. Teilung Polens auch Krakau an die Habsburgermonarchie fiel, waren in Galizien auf einmal zwei Universitäten vorhanden. Im Jahre 1802 kam der Vorschlag der Studienhofkommission, die Universität Lemberg aufzulösen und in ein Lyzeum umzuwandeln, dem Kaiser Franz I. 1805 beipflichtete (Röskau-Rydel 1999: 46). Vermöge der allerhöchsten Entschließung Franz II. ${ }^{6}$ wurden die Lemberger und die Krakauer Universität im Jahre 1805 vereinigt und in Lemberg ist ein „Liceum ${ }^{7}$ mit dem theologischen, juridischen, medicinisch-chirurgischen und philosophischen Studium ohne Facultätsrechte“ belassen worden (vgl. Handbuch des StatthaltereiGebietes in Galizien für das Jahr 1862: 153). All dies verursachte eine Unterbrechung in der Existenz der Lemberger Universität, die bis 1817 dauerte.

\footnotetext{
6 Franz Joseph Karl aus dem Hause Habsburg-Lothringen war als Franz II. noch bis 1806 Kaiser des Heiligen Römischen Reiches. Von 1804 an regierte er als Franz I. das Kaisertum Österreich.

7 Das Lyzeum bot ein zweijähriges Philosophie-Studium an, als Grundlage für die weitere Ausbildung in Rechtswissenschaften (drei Jahre), Theologie (drei Jahre) oder das zweijährige Studium am Medizinisch-Chirurgischen Institut. Dybiec (2011: 68) charakterisiert diese Einrichtung als Institution zwischen einer Mittelschule und einer Universität. Es bestand bis zur Neueröffnung der Hochschule im Jahre 1817.
} 
Nach dem Wiener Kongress von 1815 und der Entstehung der Republik Krakau (Freie Stadt Krakau) entstand erneut der Bedarf nach einer Universität in Galizien, so dass das erwähnte Lemberger Lyzeum mit der Entschließung des Kaisers Franz I. vom 7. Juli 1817 erneut zu einer Universität (unter dem Namen der K. K. Franzens-Universität) mit drei Fakultäten - der philosophischen, der theologischen und der juristischen (Röskau-Rydel 1999: 46) - erhoben und mit anderen Universitäten der Monarchie gleichgestellt wurde. Zusätzlich wurde das so genannte „medizinisch-chirurgische Studium“ eingerichtet, in dem niedere Ärzteberufe, Chirurgen und Hebammen ausgebildet wurden (Wolczanski 2011: 85; auch Dybiec 2011: 68f.). Dieser Franciszeischen Gründung war dann eine längere Existenz beschert als den Vorgängerinnen, nämlich bis zum Zerfall der Monarchie 1918.

\section{Sprachenfrage und Sprachengebrauch an der Universität Lemberg nach 1772}

Bezüglich des Sprachengebrauchs an der Universität Lemberg unmittelbar nach der Angliederung Galiziens an die Habsburgermonarchie 1772 lässt sich aufgrund historischer Dokumente annehmen, dass die Mehrheit der Vorlesungen nach wie vor in lateinischer Sprache stattfand. Dies ändert sich auch nicht sofort mit der Neugründung der Hochschule durch Joseph II. im Jahre 1784, obwohl die Verbreitung des Deutschen als Bildungssprache ein zentraler Grundsatz der Josephinischen Sprachenpolitik war.

Wie es Schiewe feststellt, war die „Sprachenpolitik von Seite des Staates“ darauf gerichtet, die Institution Universität, die allmählich ihre Autonomie verlor, in eine „Staatsanstalt“ zu verwandeln (vgl. Schiewe 1996: 259). Am 12. Juli 1784 erfolgte das kaiserliche Hofdekret (veröffentlicht im Gesetzblatt vom 29. Juli 1784), dessen Anliegen es war, in allen Bildungseinrichtungen verstärkt die deutsche Unterrichtssprache einzuführen:

Mit Anfang des nächsten Schuljahrs soll die deutsche Sprache zu den öffentlichen Vorlesungen gebraucht und eingeführt werden. Dabei ist folgende Vorschrift zu beachten:

Bei dem theologischen Studium mag der Vortrag in lateinischer Sprache zwar gegenwärtig noch fortdauern, es mögen auch die lateinischen Vorlesebücher ferner beibehalten, die Pastoraltheologie aber muss gleich in deutscher Sprache gelehrt werden.

Bei dem Juridischen sind alle Gegenstände deutsch zu lehren, nur das geistliche Recht für itzt noch ausgenommen, wobei der lateinische Vortrag und das lateinische Vorlesebuch deßwegen gestattet wird, weil alle theologische Schüler, die der deutschen Sprache nicht immer mächtig sind, es zu hören verbunden werden. In deutscher Sprache sind zwar auch die Prüfungen für das Doktorat vorzunehmen, die öffentlichen Disputazionen abzu- 
halten und die Abhandlungen für den Gradus zu verfassen; doch mag den Kandidaten noch einige Zeit die Wahl der deutschen oder lateinischen Sprache freigelassen werden.

Bei dem medizinischen wird der medizinische praktische Unterricht für Wundärzte und die Lehre der Wundarznei und Hebammenkunst ehehin schon in deutscher Sprache vorgetragen.

Bei dem philosophischen hat der Gebrauch der deutschen Sprache sich über alle Lehrgegenstände zu erstrecken. (Hofdecret vom 12. Juli 1784, zitiert nach Fischel 1910: 32f.)

Mit seinem Sprachedikt wollte Joseph II. der Tatsache Rechnung tragen, dass nach der Auflösung des Jesuitenordens bei den Studienkandidaten die Lateinkenntnisse stark zurückgingen. Zudem war an die Sprachenfragen auch der „Funktionswandel der Universität von einer relativ autonomen Korporation zu einer staatlich kontrollierten Bildungsanstalt“ gekoppelt (Schiewe 1996: 181 und 220; auch 277).

Von der sprachlichen Regelung gemäß dem zitierten Hofdekret war die Universität zu Lemberg vorerst ausgenommen (vgl. Eder 2006: 160). So schreibt Bieberstein: „An den Universitäten beginnt die Ablösung der lateinischen Vorlesungssprache durch die deutsche 1784, allerdings nicht überall: Die in diesem Jahr gegründete Universität Lemberg erhält die lateinische Vorlesungssprache“ (1993: 242; insbesondere für Naturwissenschaften s. auch Wróblewski 2011: 271). Finkel erwähnt lediglich, dass im Fach Medizin einige Vorträge auf Deutsch und Polnisch gehalten wurden (Finkel \& Starzyński 1894: 41-43). Ende des 18. Jahrhunderts dominierte eindeutig das Lateinische als Unterrichtssprache an der Universität Lemberg.

Im Detail können wir die Situation auf der Grundlage der Vorlesungsverzeichnisse analysieren. Das älteste mir verfügbare Vorlesungsverzeichnis der Universität Lemberg stammt aus dem Jahre 1784/1785. Es wurde in den „Schlözer’s Stats-Anzeigen“, Heft 36, Band IX veröffentlicht (gedruckt 1786 bei Vandenhoeks in Göttingen). Auf den Seiten 456-460 des genannten Heftes findet sich der „LectionsCatalogus von Lemberg: Praelectiones tam ordinariae quam extraordinariae in Universitate Leopoliensi, a 17 Novembr. 1784 usque ad 7 Sept. 1785 habendae. Leopoli, typis Thomae Piller, ArchiEpisc. Typographi. Fol. 4 Seiten“.

Die Beschreibungssprache des Vorlesungsverzeichnisses ist durchgehend Latein. Um welche Unterrichtssprache es sich jeweils handelte, wird in dem Vorlesungsverzeichnis kaum vermerkt. Man kann jedoch davon ausgehen, dass die Vorlesungen - mit vereinzelten Ausnahmen - auf Lateinisch gehalten wurden; dies geht oft aus dem Titel der Vorlesung hervor. Aus dieser Quelle (s. Grafik 1) wird ersichtlich, dass der Unterricht in folgenden Abteilungen erfolgte: Theologie, Jura, Medizin und Philosophie.

Im theologischen Studium gab es 1784/85 insgesamt 10 lateinische Vorlesungen wie etwa „Historia ecclesiastica“, „Encyclopaedia theologica“, „Hermeneuti- 


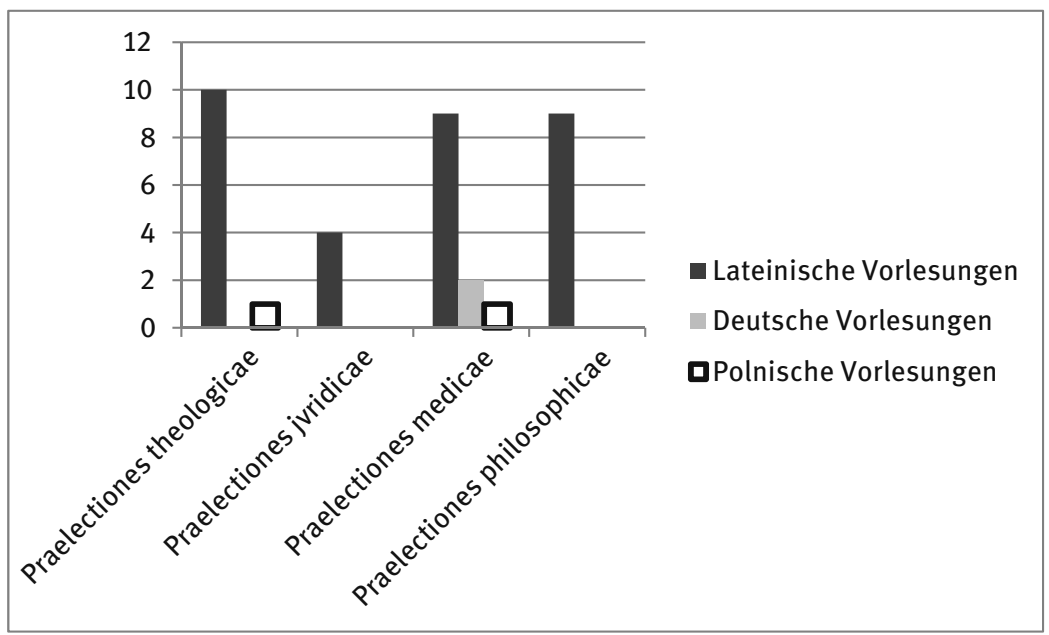

Grafik 1: Vorlesungssprachen an der Universität Lemberg im Studienjahr 1784/1785.

ca“, „Lingua graeca“, „Patrologia“, „Theologia polemica“ etc. Das theologische Studium beinhaltete eine einzige nichtlateinische Vorlesung, nämlich in Pastoraltheologie. Dieses Fach lehrte Skorodyński in polnischer Sprache, was im Vorlesungsverzeichnis auch explizit vermerkt ist (,lingua polonica“).

Das juridische Studium erfolgte 1784/85 ausschließlich in lateinischer Sprache; angeboten wurden „Jus naturae“, „Jus civile“, „Jus ecclesiasticum publicum \& privatum“, „Scientia politica“ etc. Im Rahmen des Medizinstudiums wurden Vorlesungen in Klinischer Praxis, Physiologie, Anatomie, Chemie u. a. auf Latein gehalten. Auf Deutsch erfolgte lediglich der Unterricht in Chirurgischer Physiologie („Physiologia Chirurgis, idiomate germanico hora 10 explanabitur in Auditorio medico, a D. Kriegl, Prof. extraord.“). Auch die Hebammenkunst stellte eine Besonderheit dar: Unterrichtet wurde das Fach auf Polnisch und auf Deutsch („Artem obstetriciam, germanico \& polonico idiomate, in Theatro anatomico [...] explicabit D. Waltz“).

Das Philosophiestudium enthielt ausschließlich lateinische Vorlesungen, darunter Logik, Theoretische und experimentelle Physik, Allgemeine Geschichte, Philologie und Ästhetik u.v.m. Dies bildet Grafik $1^{8}$ ab.

Zusammenfassend lässt sich festhalten, dass das Philosophie- und das Jurastudium im Jahre 1874/75 ausschließlich in Latein abliefen. Die Mehrheit der

8 Hier und in nachfolgenden Diagrammen steht die Zahl an der linken Achse für die Anzahl der wöchentlichen Lehrveranstaltungen. 
Vorlesungen der theologischen und der medizinischen Fakultät erfolgten ebenfalls in lateinischer Unterrichtssprache. Einige wenige Ausnahmen stellten die Fächer Physiologie und die Hebammenkunst im Bereich der Medizin dar, für die die deutsche Vorlesungssprache verwendet wurde. Für die Pastoraltheologie war die polnische Sprache üblich.

Gemäß der josephinischen Sprachenpolitik sollte in Lemberg der Wechsel zum Deutschen angestrebt werden, was allerdings nur sehr langsam erfolgte. 1788/89 gab es an der hiesigen Universität explizite Bemühungen, an der philosophischen Fakultät die deutsche Unterrichtssprache einzuführen (z. B. von Prof. Zehnmark), jedoch blieben diese erfolglos. Für diese und andere Fakultäten blieb zunächst das Lateinische die Unterrichtssprache. Leider stehen mir die Vorlesungsverzeichnisse aus den späteren Semestern der „Josephinischen“ Universität, die bis 1805 bestand, nicht zur Verfügung, weshalb ausführlichere Aussagen an dieser Stelle nicht möglich sind. Mit Dybiec ließe sich behaupten, dass Latein als Unterrichtssprache zunächst seine Dominanz behielt. Lediglich in der Medizin unterrichtete man - wie schon 1784/85 - sehr bald auf Polnisch und Deutsch, denn dieses Fach wurde vor allem von jüdischen Studierenden besucht (vgl. Dybiec 2011: 68f.).

\section{Unterrichtssprachen an der Franziszeischen Universität nach 1817}

Die neue, 1817 wiedergegründete Franziszeische Universität bot neben Latein verstärkt die deutsche Unterrichtssprache an. Stopka (2011: 232) stellt für die Zeit nach 1817 (bis zur Polonisierung der Universität im Jahre 1871) fest, dass an der Lemberger Universität viele deutsche Professoren mit Abschlüssen deutschsprachiger Universitäten wie Wien, Berlin, Breslau oder Göttingen dozierten. Laut Röskau-Rydel (1999) war der Lehrkörper der Lemberger Hochschule zwischen 1784 und 1848, insbesondere an der philosophischen und juristischen Fakultät, mehrheitlich aus deutschsprachigen Professoren und Wissenschaftlern zusammengesetzt, die aus Böhmen, Mähren und anderen deutschen Ländern stammten (Röskau-Rydel 1999: 47). Dies war eine wichtige Voraussetzung für den Übergang zur deutschen Unterrichtssprache.

Bestätigt wird dies auch durch einen Blick in das Vorlesungsverzeichnis der Universität Lemberg aus dem Jahre 1817, welches nur in handschriftlicher Fassung in den Beständen des Gebietsarchivs in Lviv erhalten ist. Die in handschriftlicher Form verfügbare „Ordnung der öffentlichen Vorlesungen“ aus dem Jahre 1817 gibt an, dass die damalige Universität folgende Studiengänge anbot: ein vierjähriges theologisches Studium, ein vierjähriges juridisch-politisches 
Studium, ein dreijähriges philosophisches Studium und ein zweijähriges medizinisch-chirurgisches Studium.

Im theologischen Studium lehrte man primär in lateinischer Sprache: „Kirchengeschichte“ (Franz Xaver Zachariewicz), „Das Alte Testament“ (Radkiewicz), „Dogmatik und Polemik“ (Hryniewiecki), „Über Moral“ (Joseph Jarina) u. a. In polnischer Sprache hielt Kajetan Zmigrodzki seine Vorlesung in Pastoraltheologie (für den dritten Jahrgang). Auf Deutsch wurde im Bereich Theologie kein Unterricht angeboten.

Im philosophischen Studium überwog ebenfalls Latein, mit Vorlesungen „Über theoretische Philosophie“ (Nikolaus Napadiewicz), „Über reine Mathematik“ (Franz Kodesch), „Religionswissenschaft“ (Josef Jarina), „Über griechische Philologie nach Lectiones graecae“ (Ignaz Pollak), „Physik und angewandte Mathematik“ (Anton Gloismann), „Über griechische Philologie“ (Ignaz Pollak) u. v. m.

Auf Deutsch lehrten hier in diesem Jahr Joseph Mauß („Über Universalgeschichte, nach eigenen Schriften“ und „Über die Geschichte der Österreichischen Staaten“) sowie Franz Diewald („Über allgemeine Naturgeschichte nach eigenen Schriften“). Dennoch war die deutsche Unterrichtssprache nur minimal vertreten; auf Polnisch gab es gar keine Philosophie-Vorlesungen.

Anders gestaltete sich der Sprachengebrauch im juridisch-politischen Studium: Eine ganze Reihe Vorlesungen wurde auf Deutsch gehalten, etwa „Die europäische Staatskunde“, „Die Statistik“, „Politische Wissenschaften“ und „Österreichische Gesetzkunde“ (die beiden letzteren von Josef Rohrer). Auf Polnisch wurden an dieser Fakultät keine Vorlesungen angeboten, hingegen in der lateinischen Sprache eine ganze Menge, z. B. „Die Encyklopädische Übersicht der Rechtswissenschaft“, „Das allgemeine Staatsrecht“, „Das natürliche und europäische Völkerrecht und das österreichische Kriminalrecht“, „Über das römische Recht“, „Das Kirchenrecht“, „Das Österreichische bürgerliche Recht, nach dem allg. Bürgerl. Gesetzbuch Wien 1811“, „Das ehemalige pohlnische Recht“ u. v. a.

Im medizinisch-chirurgischen Studium, das zu diesem Zeitpunkt zwei Jahre dauerte und angewandt-praktisch ausgerichtet war, fehlte das Lateinische als Unterrichtssprache hingegen komplett. Die Vorlesungen fanden mehrheitlich auf Deutsch statt: „Die Einleitung in das Chirurgische Studium und in die Anatomie“ (Stecher von Sebenitz), „Physiologie und allgemeine Pathologie“ (Zerdzynski), „Die Theoretische Chirurgie“ (Benedikt Wagner), „Die spezielle Therapie der innerlichen Krankheiten“ (Babel von Fronsberg), „Die gerichtliche Arzneykunde“ (Zerdzynski), „Die Geburtshilfe“ und „Die Thierarzneykunde“. Auf Polnisch bot Stecher von Sebenitz den Unterricht in Geburtshilfe an, und zwar nach einem ins Polnische übersetzten Lehrbuch und nach eigenen Schriften (vgl. Grafik 2). 


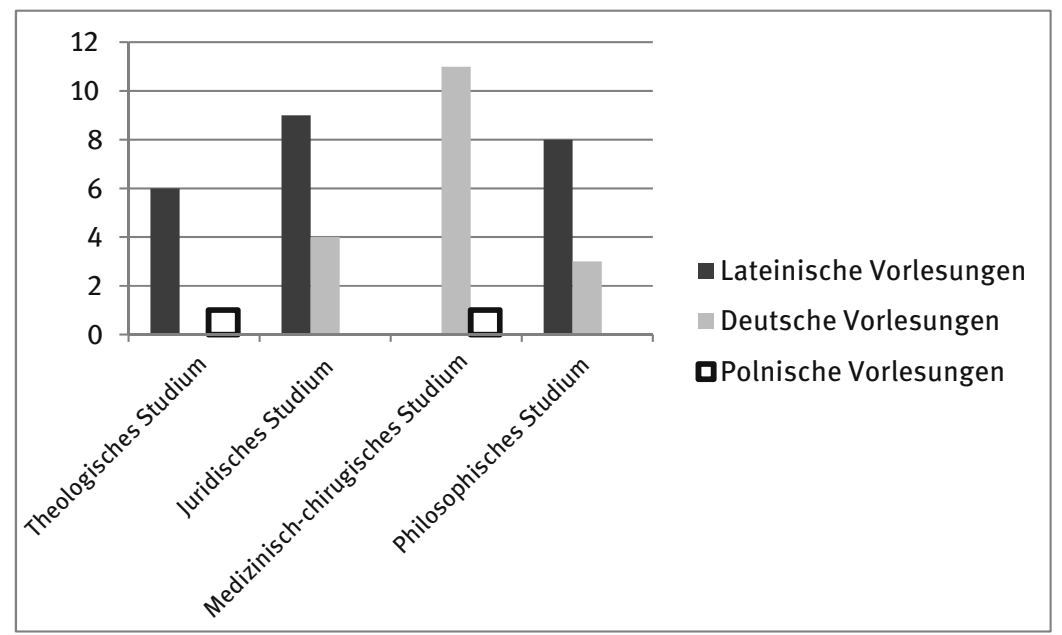

Grafik 2: Sprachengebrauch in den Vorlesungen im Jahre 1817.

Wie Diagramm 2 deutlich macht, herrschte im Jahre 1817 in der Theologie nach wie vor das Lateinische vor, mit Ausnahme der Pastoraltheologie, die in der „Volkssprache“ (auf Polnisch) unterrichtet wurde. Klare Überhand hatte das Lateinische auch in der philosophischen Fakultät. An der juridischen Fakultät waren sowohl Latein als auch Deutsch in bedeutendem Umfang vertreten.

Deutlich anders als in der ,Josephinischen“ Zeit stellte sich nun die Situation in der Lemberger Medizin dar: Hier wurde vorwiegend auf Deutsch unterrichtet, lediglich im Fach Geburtshilfe wurde Unterricht auf Polnisch angeboten. Das Lateinische verschwand aus dem medizinischen Unterricht.

Unverkennbar ist die Tendenz zur Verbreitung der deutschen Unterrichtssprache, die jedoch nicht schlagartig, sondern stufenweise erfolgt. Röskau-Rydel (1999) ist der Meinung, dass der Sprachenwechsel zum Deutschen mit der „Einführung des neuen Schulplans im Jahre 1824, der bis 1848 gültig blieb“, erfolgte; diesem Plan gemäß „wurde die deutsche Sprache in sämtlichen Fächern eingeführt, mit Ausnahme der griechischen Philologie, die in lateinischer Sprache gelehrt wurde“ (Röskau-Rydel 1999: 47). Diese Feststellung ist jedoch zu revidieren. Die ausgewerteten Vorlesungsverzeichnisse aus den Jahren $1826^{9}$ bis 1846 zeigen folgendes Bild: An der juridischen, medizinischen und philosophischen Fakultät dominieren tatsächlich die deutschsprachigen Vorlesungen (s. Grafik 3 und 4); lediglich über klassische Literatur und

9 In dem Vorlesungsverzeichnis für das Jahr 1826 wird die Vorlesungssprache explizit angegeben. 


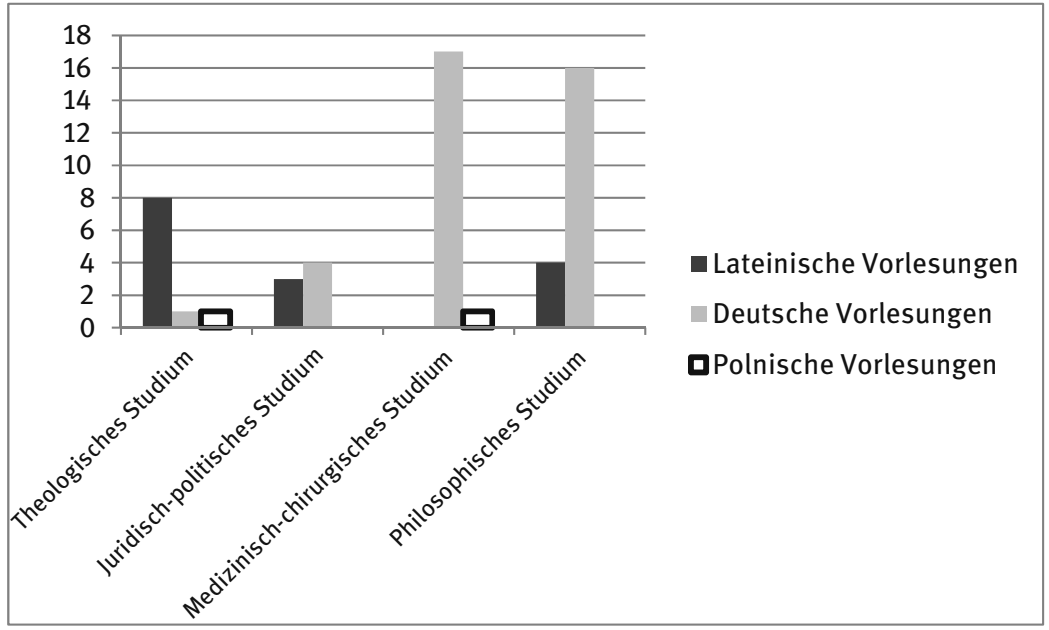

Grafik 3: Ordentliche und außerordentliche Vorlesungen an der Universität Lemberg im Jahre 1826.

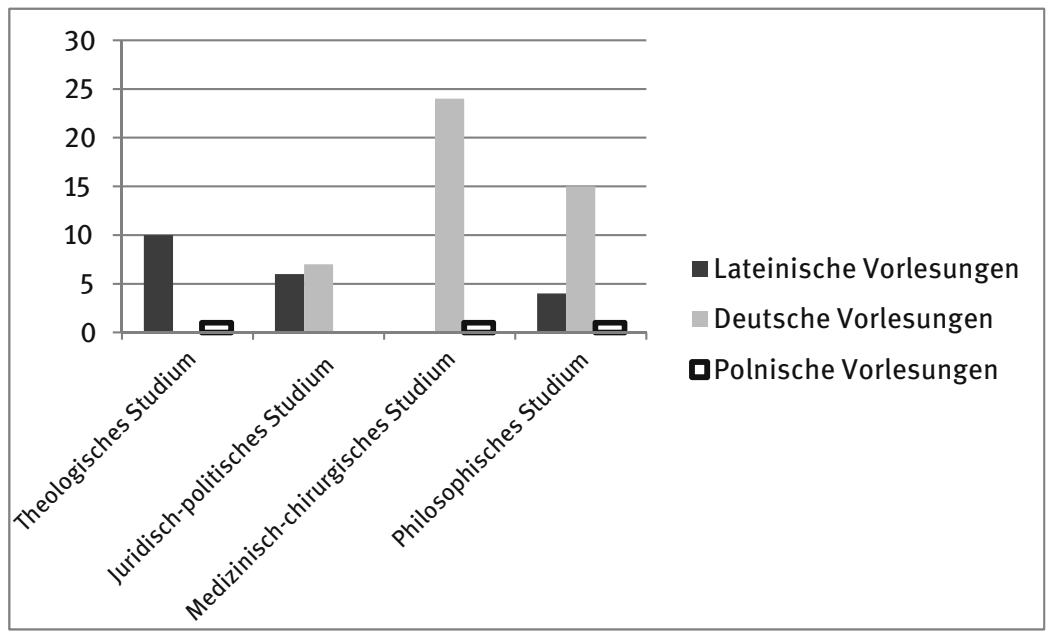

Grafik 4: Ordentliche und außerordentliche Vorlesungen an der Universität Lemberg im Jahre 1840. 
griechische Philologie wird auf Lateinisch gelehrt. Aber die Theologie bleibt nach wie vor fest „in lateinischer Hand“, mit Ausnahme der Pastoraltheologie, wo - wie auch schon in den Jahrzehnten zuvor - das Polnische und Ruthenische zugelassen sind. Somit kann man die Phase zwischen 1817 und 1826 nur mit großer Einschränkung als die Phase des Sprachenwechsels vom Lateinischen zum Deutschen für die Universität Lemberg bezeichnen.

In den Tabellen 1 und 2 sind exemplarisch Fächer der jeweiligen Fakultät nach ihren Vortragssprachen gelistet. Diese Daten geben uns einen Einblick in die Sprachwirklichkeit an der Hochschule in den Jahren 1826 und 1840. 


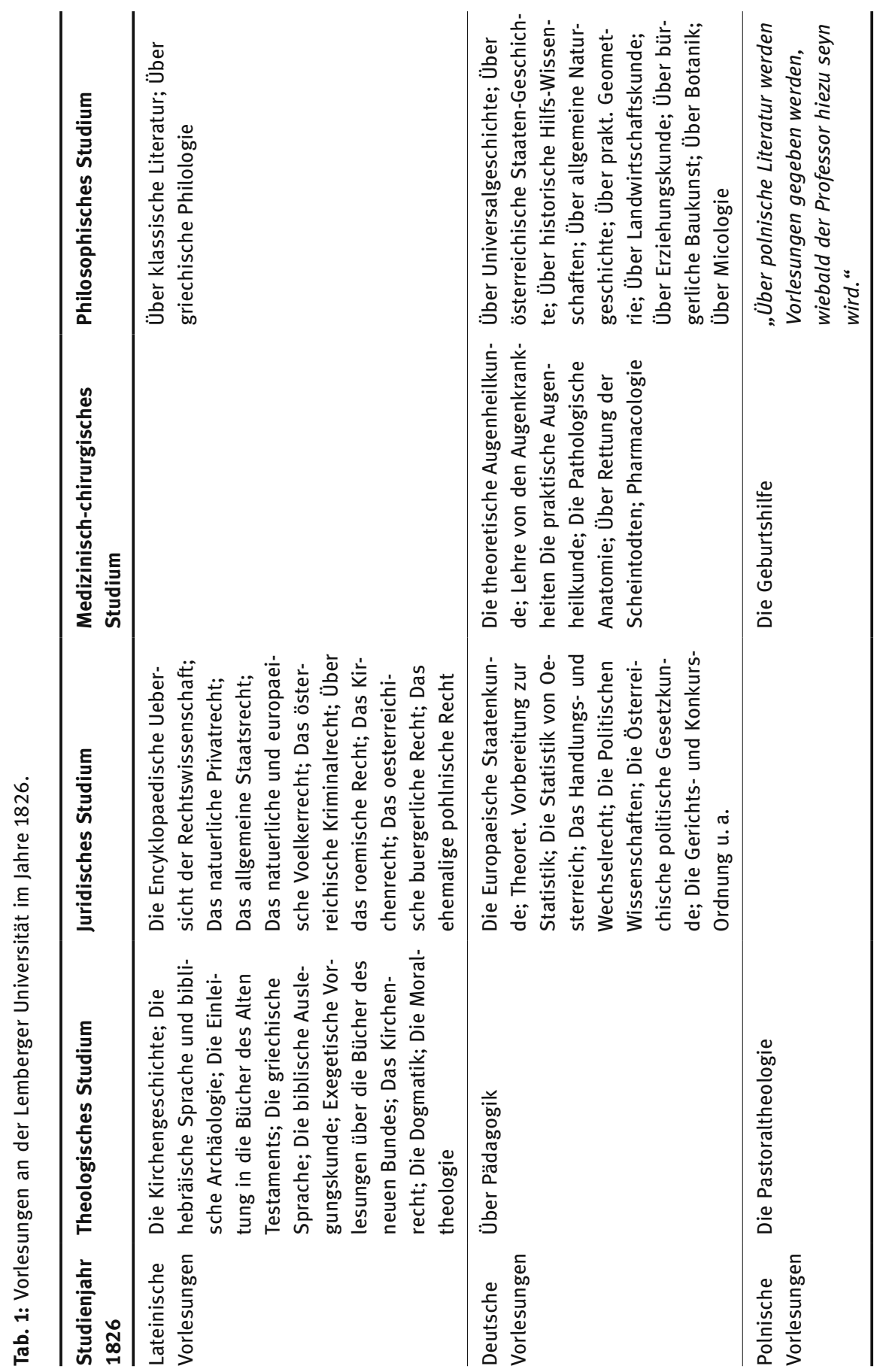




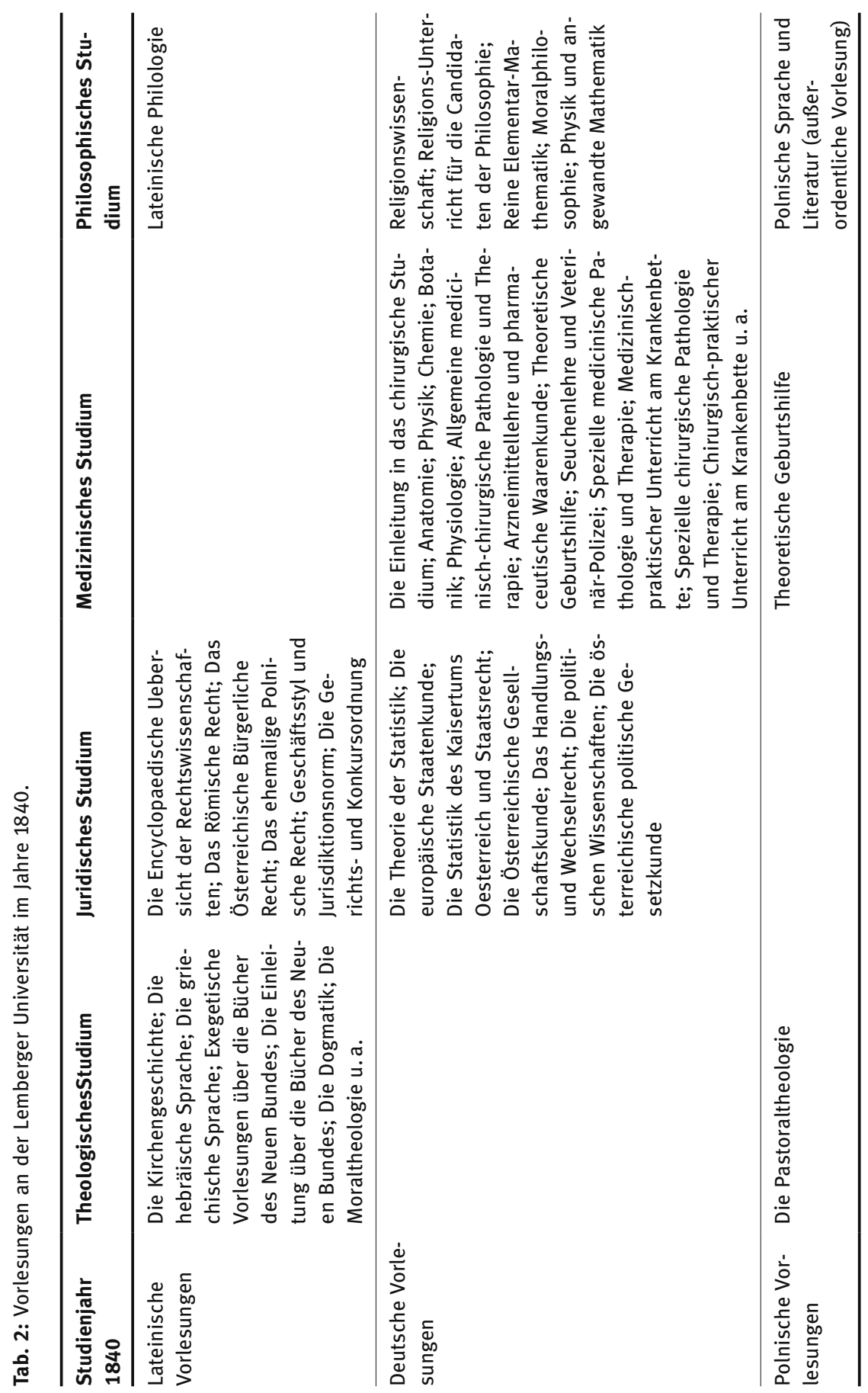


Sowohl im Jahre 1826 als auch im Jahre 1840 dominierte Deutsch im juridischpolitischen Studium, in der Medizin sowie in der Philosophie. Im Vorlesungsverzeichnis von 1840 findet sich zudem auf S. 11 - das philosophische Studium betreffend - folgende Fußnote:

Zur Lehrsprache in den gesammten Gegenständen der philosophischen Studien ist durch den $\S 31$ des mit allerhöchsten Entschließung vom 28. September 1834 genehmigten philosophischen Lehrplans die deutsche Sprache vorgeschrieben, mit Ausnahme der lateinischen Philologie, über welche der Vortrag in lateinischer Sprache gehalten wird.

Auf Polnisch wurden an der philosophischen Fakultät die Fächer Polnische Sprache und Polnische Literatur angeboten; der Besuch dieses Unterrichts war für Hörer anderer Fakultäten offen. So lesen wir auf S. 7:

Zugleich werden die Herren Juristen eingeladen, den Vorlesungen [...] über die polnische Sprache, da ihnen der Beweis hierüber bei dem künftigen Eintritte in öffentliche Dienste nothwendig ist, beizuwohnen.

Für das Jahr 1846 lässt sich ein fast identisches Fächerangebot nachweisen (siehe das entsprechende Vorlesungsverzeichnis); die sprachlichen Verhältnisse bleiben unverändert. Das Ruthenische kommt im universitären Unterricht bislang nicht vor.

Bis zum Völkerfrühling bleibt die sprachliche Situation hinsichtlich der Vorlesungen an der Universität Lemberg unverändert. Während an der theologischen und juridischen Fakultät mehrheitlich in lateinischer Sprache gelehrt wird, dominiert in der Medizin die deutsche Unterrichtssprache. Dasselbe gilt für die philosophische Fakultät, nicht zuletzt aufgrund der Vorschrift der deutschen Unterrichtssprache gemäß dem bereits erwähnten $\S 31$, mit Ausnahme der lateinischen Philologie. Bis dahin gibt es nur wenige Fächer auf Polnisch zu hören, dazu gehören die praktische Geburtshilfe oder die Pastoraltheologie. Das Ruthenische war bis zum Völkerfrühling im Universitätsbetrieb kaum vertreten. Die Verwaltungssprache ist hingegen überwiegend Deutsch. Insgesamt auf die erste Hälfte des 19. Jahrhunderts blickend ließe sich behaupten, dass bis 1848 kein Sprachwechsel stattgefunden hat. Dennoch gab es deutliche Verschiebungen im Sprachgebrauch an der Universität, die zum Teil durch Bestrebungen der lokalen Eliten verursacht waren, jedoch zum größeren Teil durch sprachenpolitische Bestimmungen „von oben“ (wie etwa der bereits erwähnte $\S 31$ u. v. m.) stattgefunden haben. 


\section{Sprachengebrauch nach 1848: Umbrüche und Kontinuitäten}

Der „Völkerfrühling“ 1848 brachte für die österreichische Monarchie im Ganzen wie auch für Galizien im Besonderen einige Gesetzesentwürfe hervor, die die Gleichberechtigung der landesüblichen Sprachen im Fokus hatten (vgl. Ptashnyk 2013: 99f.). Dieses Jahr markierte auch den Beginn einer neuen Phase in der Geschichte der Universität (Dybiec 2011: 69), die durch Kämpfe um die Unterrichtssprache geprägt war.

Eine der Neuerungen, die die Ereignisse von 1848 mit sich brachten, war die vermehrte Einführung des Polnischen und teilweise des Ukrainischen als Unterrichtssprachen an der Universität. Unter dem Innen- und Bildungsminister Franz Seraph Stadion (1848/49) wurden Schritte zur „Nationalitätsberuhigung “ unternommen:

Die Universitäten erhielten das Recht, in den jeweiligen Landessprachen zu unterrichten, und gewichtige, sich als national bekennende Persönlichkeiten - wie etwa František Ladislav Čelakovský, Jakiv Holovac'kyj, Wincenty Pol und Jan Evangelista Purkyné - wurden als Lehrkräfte berufen. (Surmann 2009: 16)

Bis 1848 fanden das Ukrainische und das Polnische - wie bereits erwähnt verhältnismäßig wenig Anwendung als Unterrichtssprache der Universität Lemberg, wie aus den ausgewerteten Vorlesungsverzeichnissen ersichtlich ist. 1778 wurde zwar per Imperialerlass das Ruthenische als Unterrichtssprache im Fach Theologie zugelassen, es konnte sich aber an der Universität kaum etablieren. Am 19. Dezember 1848 wurde der Lehrstuhl für ukrainische Sprache und Literatur (mit Jakiv Holovac'kyj an der Spitze) eröffnet. Diese - wie Surmann schreibt - „symbolische Berufung“ von Holovac'kyj belegt, dass sich „die Politik des Statthalters Agenor Gołuchowsky, der eine Spaltung Galiziens in zwei Landesteile befürwortete, durchsetzen konnte“ (Surmann 2009: 16). Auch in der neuabsolutistischen Zeit bestand weiterhin die Lehrkanzel für Ruthenische Sprache und Literatur, ungeachtet erneuter Germanisierungstendenzen.

Der Lehrstuhl für Polnische Sprache und Literatur mit polnischer Unterrichtssprache wurde schon 1817 eröffnet. Zudem gab es einen Lehrstuhl für polnisches Recht (seit 1811) sowie einen Lehrstuhl für Slawische Literaturen (Sirka 1980: 139; auch Dybiec 2011). Unter dem Druck der polnischen Eliten verordnete die Wiener Regierung am 29. September 1848 sogar die Einführung der polnischen Unterrichtssprache an der gesamten Universität. Die Revolutionsereignisse, gepaart mit den Protesten der deutschsprachigen Professoren, verhinderten jedoch die Umsetzung dieser Verordnung an der Universität Lem- 
berg, sodass am 4. Dezember 1848 eine neue Regelung erfolgte, die die deutsche Unterrichtssprache an der gesamten Universität anordnete (Dybiec 2011: 69).

In der Epoche des Neoabsolutismus ${ }^{10}$ (bis ca. 1860) wurde die sog. nationale Frage wieder außer Acht gelassen. Es bot sich keine Möglichkeit, sprachbezogene Forderungen zu stellen (Finkel \& Starzyński 1894: 4). Ganz im Gegenteil: Das Jahr 1853 gilt in der Geschichte als das Jahr der gewaltsamen Germanisierung der Universitäten. Dabei kommt Krakau und Lemberg eine besondere Stellung zu, denn diese waren die einzigen Universitäten, an denen „die Germanisierung mit einem Kaiserlichen Erlass durchgesetzt wurde“ (Surmann 2009: 17).

Das Vorlesungsverzeichnis für das Wintersemester 1855/56 ergibt folgendes Bild der Sprachverhältnisse an der Universität Lemberg, inkl. der Medizinischchirurgischen Lehranstalt:

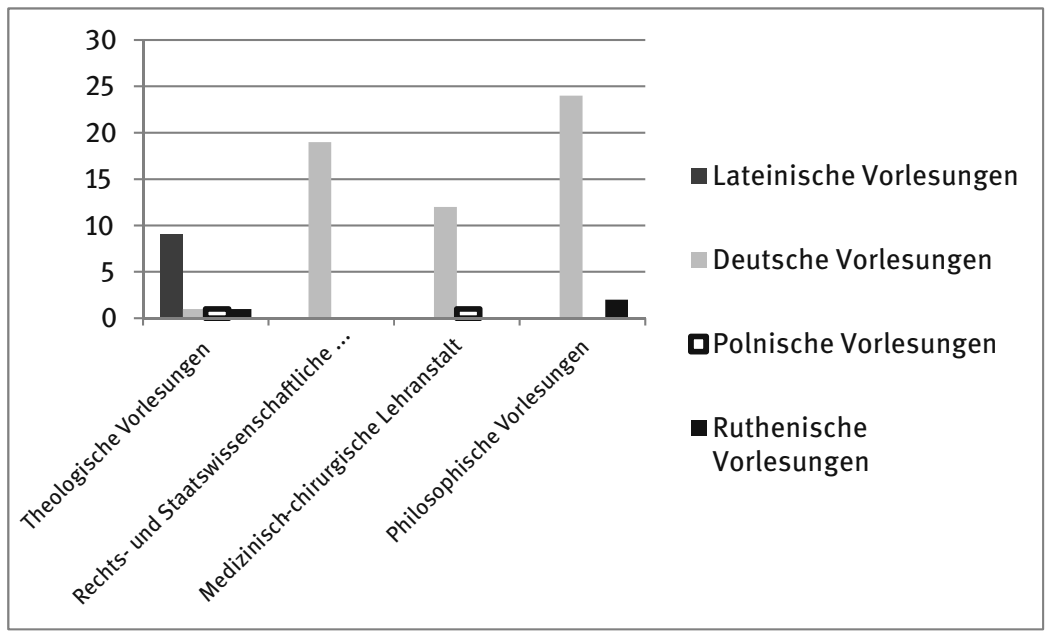

Grafik 5: Unterrichtssprachen in Wintersemester 1855/56, in absoluten Zahlen.

Während die Theologie nach wie vor von der lateinischen Unterrichtssprache dominiert wird, ist in anderen Fächern das Deutsche die überwiegende Unterrichtssprache. Ruthenisch ist nur für die speziellen philologischen Fächer vor-

10 Am 31. Dezember 1851 gab Franz Joseph I. sein „Silvesterpatent“ heraus und setzte damit die Oktroyierte Märzverfassung außer Kraft. Damit wurde die absolute Monarchie wieder eingeführt. Das zuvor so häufig postulierte Prinzip der Gleichberechtigung der Nationalitäten blieb in diesem Patent unerwähnt. 
gesehen - die Ruthenische Sprache und Literatur. Auf Polnisch konnte man an der Medizinisch-chirurgischen Lehranstalt das Fach Theoretische Geburtshilfe hören. Bis 1870 hatte die Universität Lemberg den Charakter einer deutschen Universität, so Sirka (1980: 138).

\section{Statt eines Ausblicks: Die Sprachensituation in den 1870er Jahren}

Am 4. Juli 1871 wurde durch kaiserliche Entschließung an der Universität Lemberg „die alleinige polnische Vortragssprache eingeführt“ (vgl. Strakosch-Grassmann 1905: 284), wodurch die verpflichtende deutsche Unterrichtssprache abgeschafft wurde. Polnisch und Ukrainisch wurden als Unterrichtssprachen anerkannt. Ruthenisch kam jedoch aus verschiedenen Gründen noch sehr lange kaum zum Zuge. In den 1870er Jahren wurde die Universität Lemberg praktisch $\mathrm{zu}$ einer polnischsprachigen Institution. Deutsche Professoren wurden durch Polen ersetzt, die sowohl aus Galizien als auch aus dem russischen oder preuBischen Polen kamen (Sirka 1980: 140).

Als Veranschaulichung möchte ich ein Vorlesungsverzeichnis aus den 1870er Jahre zitieren: Die „Ordnung der Vorlesungen an der k. k. Universität zu Lemberg im Sommer-Semester des Studienjahres 1875/76“ ergibt folgendes Bild: An der theologischen Fakultät zählt man in diesem Jahr 16 Vorlesungen in lateinischer Sprache und eine Vorlesung auf Deutsch („Erziehungs-Wissenschaft“). Für die Fächer „Catechetica et Methodica sowie „Theologia Pastoralis“ ist jeweils eine Vorlesung in „lingua ruthena“ und eine in „lingua polona“ vorgesehen.

An der rechts- und staatswissenschaftlichen Fakultät überwiegt die polnische Unterrichtssprache (28 Vorlesungen); auf Deutsch werden sechs Vorlesungen gehalten; die ruthenische Unterrichtssprache gibt es in zwei Vorlesungen. An der philosophischen Fakultät wird fast ausschließlich auf Polnisch gelehrt (55 Veranstaltungen); daneben gibt es 4 Vorlesungen auf Ruthenisch (s. Grafik 6).

Als Vortragssprache überwiegt insgesamt zweifelsohne das Polnische. Das Vorlesungsverzeichnis an sich ist noch überwiegend in deutscher Beschreibungssprache verfasst, ${ }^{11}$ jedoch nicht durchgehend: Das Dokument zeigt span-

11 Auch dies ändert sich Ende der 1870er Jahre, ab 1879 liegen für die Universität Lemberg nur noch polnischsprachige Vorlesungsverzeichnisse vor, die semesterweise unter dem Titel „Skład Uniwersytetu i Program wykładów“ bis 1918 erscheinen. 


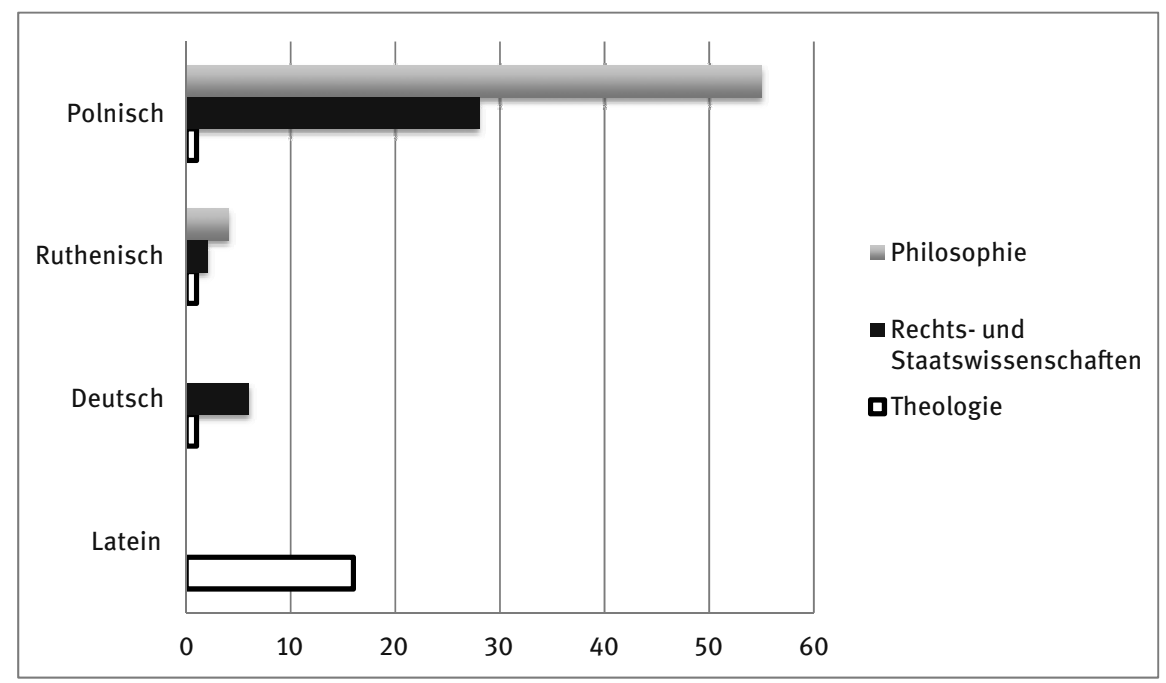

Grafik 6: Sommer-Semester des Studienjahres 1876: Vorlesungen in der jeweiligen Unterrichtssprache (in absoluten Zahlen). ${ }^{12}$

nende Beispiele für Code-Switching, da die Vorlesungstitel in der jeweiligen Vortragssprache angegeben sind (Ukrainisch, Polnisch, Deutsch und Latein).

Im ausgehenden 19. Jahrhundert besteht in Lemberg eine weitgehend polonisierte Universität. Einige andere Sprachen sind zwar auch noch vertreten, aber bei weitem nicht mehr in dem Verhältnis, wie es noch zu Beginn des Jahrhunderts war. Wie stand es zu dieser Zeit um das Ruthenische als Unterrichtssprache? Nur langsam setzten die Ruthenen ihre Sprachenforderungen durch. Im ausgehenden 19. Jahrhundert intensiviert sich die Auseinandersetzung um die ruthenische Sprache an der Universität, jedoch ohne größere Erfolge. Den Wünschen nach Gründung einer separaten, ukrainischsprachigen Universität, die im Jahr 1901 artikuliert worden waren, „wurde nicht entsprochen“ (Burger 1995: 184; vgl. auch Mudryj 2002). Allerdings wurden an der Universität in Lemberg bis zum Jahr 1914 zehn Lehrkanzeln mit ruthenischer Unterrichtssprache eingerichtet (Surmann 2009: 24).

12 Die medizinische Fakultät bestand zu diesem Zeitpunkt nicht - sie wurde ausgegliedert in ein separates medizinisch-chirurgisches Studium. Deshalb liegen hierfür keine Daten vor. 


\section{Resümee}

Im ausgehenden 18. Jahrhundert ereigneten sich an Universitäten der Habsburger Monarchie wichtige Veränderungen, zu denen auch der Sprachenwechsel zur deutschen Kommunikationssprache gehörte. Letzterer lag nicht an inneruniversitären Bedürfnissen, sondern an der staatlichen Sprachenpolitik, welche die Germanisierung des Bildungswesens im Fokus hatte.

Das analysierte Quellenmaterial, vor allem die Vorlesungsverzeichnisse der Universität Lemberg aus dem ausgehenden 18. und dem 19. Jahrhundert, haben deutlich gezeigt, dass der Sprachenwechsel vom Lateinischen zum Deutschen mehrstufig und komplex verlief. Die Germanisierung der Hochschule hing stark mit der Sprachenpolitik Wiens zusammen, ganz besonders in der Phase des Neoabsolutismus. Vor allem ließ sich beobachten, dass die Praxis des Gebrauchs des Lateinischen als Vorlesungssprache an der Universität Lemberg sich als ziemlich beharrlich erwies und dass der Übergang vom Lateinischen zu den Volkssprachen von Fakultät zu Fakultät unterschiedlich verlief. So wurden im medizinischen Studium schon sehr früh Vorlesungen in der deutschen Sprache angeboten; in der Theologie verschwindet das Lateinische nicht einmal Ende des 19. Jahrhundert aus dem Vorlesungsverzeichnis. Die langjährige starke Präsenz des Lateinischen im Fach Theologie lässt sich ähnlich wie Schiewe (1996) es für die Universität Freiburg tut - dadurch erklären, dass hier die Kirche an der Kommunikation beteiligt war. Die Kommunikation der römisch-katholischen Kirche fand bekanntlich primär auf Lateinisch statt (vgl. Schiewe 1996: 210). In Lemberg kommt ein weiterer spezifischer Umstand hinzu: Die Lemberger Theologie hatte sowohl den römisch-katholischen (polnisch-, armenisch- und deutschsprachigen) als auch den griechisch-katholischen (meist ruthenischsprachigen) Klerus auszubilden. Für all diese Sprachgruppen und Konfessionen war das Latein die universale Vermittlungssprache.

Die besondere Komplexität des Sprachwechsels vom Lateinischen zum Deutschen an der Universität Lemberg ist dadurch zu erklären, dass es sich dabei nicht um den Übergang von einer Bildungs- zu einer Volkssprache handelt, wie man es von den meisten europäischen, v. a. deutschen Universitäten kennt: In Lemberg war Deutsch nur die Muttersprache eines kleinen, wenn auch einflussreichen Bevölkerungsteils. Im Grunde genommen war dort der Wechsel vom Lateinischen zum Deutschen der Wechsel von einer Bildungssprache zu einer anderen.

Der analysierte Sprachenwechsel - zunächst zum Deutschen, später zum Polnischen und teilweise Ruthenischen als Vorlesungs- und Wissenschaftssprache - war durch sehr vielfältige außersprachliche Faktoren bedingt bzw. beeinflusst. Dass sich Deutsch an der Universität Lemberg für einen längeren 
Zeitraum etablieren konnte, hängt mit seiner Funktion als Staatssprache zusammen, mit der Sprachenpolitik Wiens sowie mit der Tatsache, dass sich das Ukrainische/Ruthenische und Polnische im 19. Jahrhundert noch auf anderen Stufen der Standardisierung befanden als das Deutsche. Da nach 1848 sich die Frage nach der Gleichberechtigung aller landesüblichen Sprachen zuspitzte und später sich die meisten Sprachkonflikte entlang des polnisch-ukrainischen Verhältnisses formierten, konnte das Deutsche zunächst für eine Art Neutralität sorgen.

Das Deutsche spielte im Hochschulbereich noch länger eine Rolle als in den anderen Domänen des öffentlichen Lebens in Lemberg: Die 1860er und 1870er Jahre sind immer noch eine „Blütezeit“ für die deutsche Vortragssprache an der Universität Lemberg. In allen anderen Bereichen - etwa Mittelschulen (vgl. dazu Ptashnyk 2015: 284 f.), Gerichtswesen, Stadtverwaltung, Pressewesen - ist schon früher eine deutliche Polonisierung festzustellen. An der Universität Lemberg kam diese Entwicklung etwas später, aber das war nur eine Frage der Zeit. Von einer „Monolingualität“ an der Universität Lemberg konnte bis 1918 gar keine Rede sein. Im Grunde wurde weder eine vollständige Germanisierung noch eine vollständige Polonisierung der Lemberger Universität erreicht. In allen Epochen war ein Nebeneinander von mindestens drei Sprachen zu beobachten.

\section{Primärquellen}

Acta Vniversitatis Leopolitanae in Galicia Anno MDCCLXXXIV. Inauguratae. Leopoli, 1786. Lemberg: Druckerei von Thomas Piller.

Akademische Behörden an der K. K. Universität zu Lemberg: sammt der Ordnung der Vorlesungen an derselben. [Jahrgänge 1862 bis 1879].

Die Ruthenische Sprach- und Schriftfrage in Galizien (1861). Lemberg: Schnellpresse des Stauropigianischen Instituts.

Handbuch des Statthalterei-Gebietes in Galizien für das Jahr [...]. Lemberg: K. K. Galizische Aerarial-Staats-Druckerei [Jahrgänge 1862 bis 1866].

LectionsCatalogus von Lemberg: Praelectiones tam ordinariae quam extraordinariae in Universitate Leopoliensi, a 17 Novembr. 1784 usque ad 7 Sept. 1785 habendae. Leopoli, typis Thomae Piller, ArchiEpisc. Typographi. In: Schlözer's Stats-Anzeigen, Heft 36, Band IX/1786. Göttingen: Vandenhoeks, 456-460.

Österreichische Statistik. Hg. von der k. k. statistischen Central-Commission. Neue Folge, Band 1, Wien 1912.

Ordnung der öffentlichen Vorlesungen, welche an der seiner Kais. Majestät Franz I. allerhöchsten Namen führenden Universität zu Lemberg im Schuljahre [...] gehalten wurden [Jahrgänge 1826 bis 1879].

Retzbach, Johann Michael (Hrsg.): Taschenbuch der k. k. Lemberger Universität. Lemberg: Schnayder'sche Schriften. [Jahrgänge 1819, 1822 und 1838]. 
Spis wykładów w C. K. Uniwersytecie imienia Cesarza Franciszka I we Lwowie [...] / Program wykładów w C. K. Uniwersytecie imienia Cesarza Franciszka we Lwowie w [...] / Skład personelu i program wykładów w [...] półroczu [...]. C. K. Uniwersytet im. Cesarza Franciszka I. we Lwowie [Jahrgänge 1876 bis 1890].

\section{Literatur}

Dybiec, Julian (2011): Uniwersytet Lwowski pod zaborem austriackim. In: Wanda Lohman (Hrsg.), Universitati Leopoliensi trecentesimum quinquagesimum anniversarium suae fundationis celebranti in memoriam. Kraków: Polska Akademia Umiejętności, 66-76.

Eder, Ulrike (2006): „Auf die mehrere Ausbreitung der teutschen Sprache soll fürgedacht werden": Deutsch als Fremd- und Zweitsprache im Unterrichtssystem der Donaumonarchie zur Regierungszeit Maria Theresias und Josephs II. Innsbruck u. a.: StudienVerlag.

Finkel, Ludwik \& Stanisław Starzyński (1894): Historya uniwersytetu Lwowskiego. Lwów: Nakładem Senatu akademickiego c. k. Uniwersytetu Lwowskiego.

Fischel, Alfred (1910): Das Österreichische Sprachenrecht: eine Quellensammlung. Brünn: Irrgang.

Jaworski, Franciszek (1912): Uniwersytet Lwowski. Wspomnienie jubileuszowe. Lwów: Nakładem Tow. Mitośników Przeszłości Lwowa.

Łuszczak, Grzegorz (2011): Kierunki nauczania i kadra dydaktyczna Iwowskich szkot jezuickich 1661-1773. In: Wanda Lohman (Hrsg.), Universitati Leopoliensi trecentesimum quinquagesimum anniversarium suae fundationis celebranti in memoriam. Kraków: Polska Akademia Umiejętności, 44-63.

Mudryj (2002) = Мудрий, Мар'ян (2002): Від Австрії до Польщі. Проблема українського університету у Львови в першій чверті XX століття. In: Kazimiersz Karolczak (Hrsg.), Lwów - miasto, społeczeństwo, kultura: studia z dziejów Lwowa [= Львів - місто, суспільство, культура]. Kraków: Wydawn. Naukowe WSP, 291-310.

Ptashnyk, Stefaniya (2013): Stadtsprachen historisch betrachtet: Zur Beschreibung der Mehrsprachigkeit in Lemberg 1848-1900. In: Christoph Kolbeck, Reinhard Krapp \& Paul Rössler (Hrsg.), Stadtsprache(n) - Variation und Wandel. Heidelberg: Winter, 95110.

Ptashnyk, Stefaniya (2015): Deutsch im alten Österreich: Zur Mehrsprachigkeit und Variation im habsburgischen Bildungswesen in der 2. Hälfte des 19. Jahrhunderts am Beispiel der Stadt Lemberg. In: Alexandra N. Lenz et al. (Hrsg.), Dimensionen des Deutschen in Österreich. Variation und Varietäten im sozialen Kontext. Frankfurt a. M. u. a.: Peter Lang, 377-392.

Röskau-Rydel, Isabel (1999): Deutsche Geschichte im Osten Europas. Galizien. Berlin: Siedler.

Schiewe, Jürgen (1996): Sprachenwechsel - Funktionswandel - Austausch der Denkstile: Die Universität Freiburg zwischen Latein und Deutsch. Tübingen: Niemeyer.

Sirka, Ann (1980): The Nationality Question in Austrian education. The case of Ukrainians in Galicia 1867-1914. Frankfurt a. M. u. a.: Peter Lang.

Stopka, Krzysztof (2011): Nauki historyczne na Uniwersytecie Lwowskim. In: Wanda Lohman (Hrsg.), Universitati Leopoliensi trecentesimum quinquagesimum anniversarium suae fundationis celebranti in memoriam. Kraków: Polska Akademia Umiejętności, 225-266. 
Surmann, Jan (2009): Figurationen der Akademia. Galizische Universitäten zwischen Imperialismus und multiplem Nationalismus. In: Doktoratskolleg Galizien (Hrsg.), Galizien. Fragmente eines diskursiven Raums. Innsbruck u. a.: Studienverlag, 15-26. Wróblewski, Andrzej K. (2011): Fizyka na Uniwersytecie we Lwowie. In: Wanda Lohman (Hrsg.), Universitati Leopoliensi trecentesimum quinquagesimum anniversarium suae fundationis celebranti in memoriam. Kraków: Polska Akademia Umiejętności, 267-289. 
